\title{
Lattice statistical theory of random walks on a fractal-like geometry
}

\author{
John J. Kozak, ${ }^{1,2}$ Roberto A. Garza-López, ${ }^{3}$ and Enrique Abad $^{4}$ \\ ${ }^{1}$ DePaul University, 243 South Wabash, Chicago, Illinois 60604-2301, USA \\ ${ }^{2}$ Beckman Institute, Caltech, Pasadena, California 91125, USA \\ ${ }^{3}$ Department of Chemistry and Seaver Chemistry Laboratory, Pomona College, Claremont, California 60604-2301, USA \\ ${ }^{4}$ Departamento de Física Aplicada, Centro Universitario de Mérida, Universidad de Extremadura, E-06800 Mérida, Spain
}

(Received 5 December 2013; published 31 March 2014; corrected 3 April 2014)

\begin{abstract}
We have designed a two-dimensional, fractal-like lattice and explored, both numerically and analytically, the differences between random walks on this lattice and a regular, square-planar Euclidean lattice. We study the efficiency of diffusion-controlled processes for flows from external sites to a centrosymmetric reaction center and, conversely, for flows from a centrosymmetric source to boundary sites. In both cases, we find that analytic expressions derived for the mean walk length on the fractal-like lattice have an algebraic dependence on system size, whereas for regular Euclidean lattices the dependence can be transcendental. These expressions are compared with those derived in the continuum limit using classical diffusion theory. Our analysis and the numerical results quantify the extent to which one paradigmatic class of spatial inhomogeneities can compromise the efficiency of adatom diffusion on solid supports and of surface-assisted self-assembly in metal-organic materials.
\end{abstract}

DOI: 10.1103/PhysRevE.89.032147

PACS number(s): 05.40.Fb, 61.43.Hv, 05.50.+q

\section{INTRODUCTION}

Diffusion-reaction dynamics on substrates, heterogeneous catalysis, and diffusive transport in porous and amorphous media have been modeled by random walks (RWs) on fractal lattices (see the representative references [1-7] and work cited therein). There exists an extensive literature both on disordered (random) fractals [8-10] and deterministic fractals such as the Sierpinski gasket [11-17]. In this article, we introduce a fractal-like geometry which yields analytic results in two complementary cases: diffusion from satellite sites to a centrosymmetric trap, and diffusion from a centrosymmetric source to boundary sites. This fractal-like lattice is related to the Euclidean $d=2$ dimensional square-planar lattice as shown in Fig. 1. The physical motivation for introducing this fractal-like lattice is that the influence on reaction efficiency of such lattice imperfections as terraces, ledges, and kinks or, in minerals, grain boundaries can be studied systematically and quantified (see the representative references [18-20] and work cited therein).

In developing a lattice model to study diffusion-reaction processes on surfaces having irregularities or a distributed set of imperfections, the (restricted) geometry of the system is specified by identifying sites on the host lattice inaccessible to a diffusing particle. On the finite square-planar lattice shown in Fig. 1(a), a randomly diffusing atom or molecule has, at each interior site, access to four nearest-neighbor sites, and either two or three nearest-neighbor sites if on the boundary. Except for sites on the two $C_{2}$ axes of the lattice, i.e., the two axes intersecting at the centrosymmetric site $S$, pathways to $S$ are restricted to $d=1$ channels. Thus, for example, displacements from site 2 are restricted to sites 1 only; explicitly excluded are transitions to the two symmetry-equivalent sites 4 .

In this article we mobilize the theory of finite Markov processes (see Sec. II). An exact invariance relation derived by Montroll and Weiss for random walks on a periodic lattice of uniform coordination (or valence $v$ ) and a single deep trap [21] can be used as an acid test to assess the accuracy of the numerical results obtained in this article (Sec. III).
For the lattices displayed in Fig. 1, this invariance relation can be applied if the valence of all sites on each lattice is specified to be $v=4$. For the square-planar lattice shown in Fig. 1(a), a random walker at an interior site has access to four nearest-neighbor sites. If the RW is at a boundary site of the lattice [Fig. 1(a)], possible transitions "off" the lattice are regarded as "virtual" displacements, the random walker returning to its point of departure. With this specification, a RW at a corner site can migrate to either of two nearest-neighbor lattice sites (with two possible "virtual" displacements) or, if at any other boundary site, to three nearest-neighbor sites (with one "virtual" displacement). All sites of the lattice [Fig. 1(a)] can then be assigned a coordination number, $v=4$; for the special case of a centrosymmetric trap this assignment of $v=$ 4 for all lattice sites amounts to imposing periodic boundary conditions on the host lattice. As for the fractal-like lattice [Fig. 1(b)] there are two possible "real" displacements of a random walker at each boundary site (with two "virtual" displacements); the same prescription applies to an atom or molecule moving in a channel.

The following three sections describe the application of the theory of finite Markov processes to the two diffusion-reaction processes cited earlier. Sections V and VI present companion results for the same scenarios derived using classical, Fickian diffusion theory. The results are summarized and compared in the concluding section (Sec. VII) where their relevance to specific experimental problems is pointed out.

\section{THEORETICAL BACKGROUND}

In this article, we mobilize both the theory of finite Markov processes, and classical diffusion theory. Formal aspects of the Markovian lattice statistical theory are only summarized here; a number of excellent reviews are available (see, for example, Ref. [22] and references cited therein). To study the dynamics and long-time behavior, we proceed from the probability distribution function $\rho_{n}(t)$ describing the space-time evolution of a random walker on an $N$-site lattice. If $i$ denotes the initial state of the system, and $\rho_{n}(t)$ is the probability of the atom 
(a) regular, square-planar lattice;

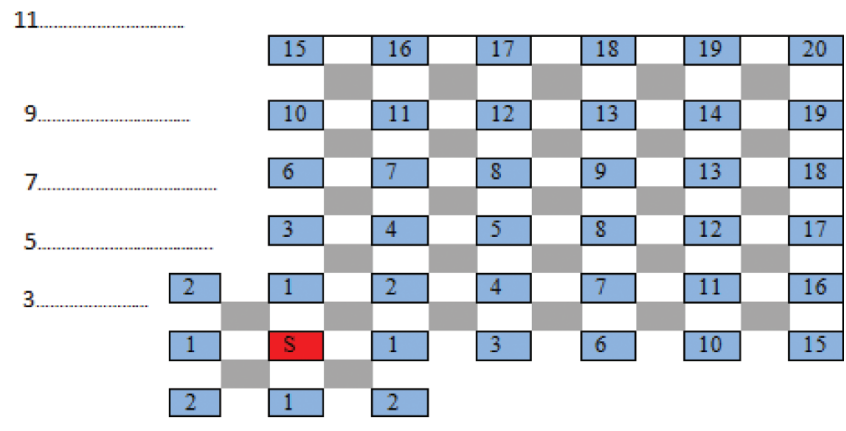

(b) fractal-like lattice.

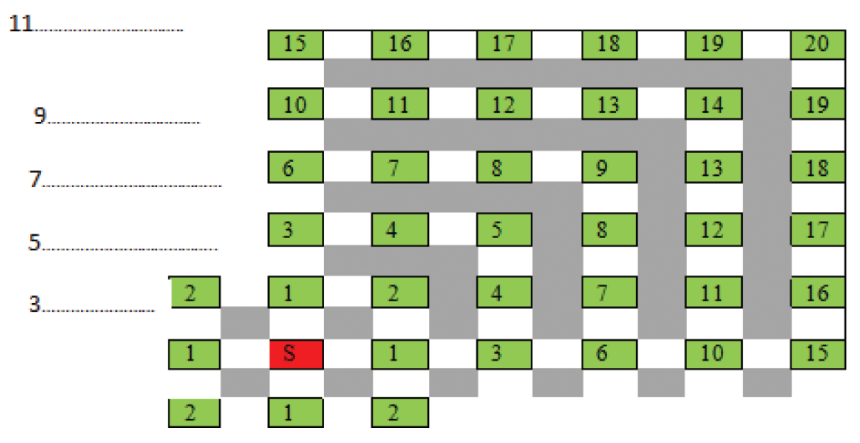

FIG. 1. (Color) Symmetry-equivalent sites on an $11 \times 11$ lattice with a centrosymmetric sink or source $S$. Nearest-neighbor displacements of the diffusing particle are permitted between spaces in white but forbidden between spaces in gray. (a) Regular, square-planar lattice; (b) fractal-like lattice. The level indices $\lambda$ corresponding to the different rows in the lattice are displayed on the left-hand side of each subfigure.

or molecule being in a specific, intermediate state $n$ at time $t$, then the stochastic master equation

$$
\frac{d \rho_{n}(t)}{d t}=-\sum_{m=1}^{N} G_{n m} \rho_{m}(t)
$$

is to be solved subject to the initial condition

$$
\rho_{m}(t=0)=\delta_{m, i} .
$$

In Eq. (1), $G_{n m}$ is the (probability) rate for a transition to the state $n$ from the state $m$. The $\mathbf{G}$ matrix is linked to the $N \times N$ Markov transition probability matrix $\mathbf{P}$ with elements $p_{n m}$ defined via the relation

$$
G_{n m}=\delta_{n, m}-p .
$$

Here, $p_{n m}$ is the probability that the random walker, conditional on being in state $m$ at time $t$, will be in the state $n$ in the next step, until the terminal state is reached. The transition probability is equal to one divided by the coordination number of the starting site; here, $p_{n m}=1 / 4$ for both lattices (Fig. 1).

In what follows, we calculate the mean number $\langle n\rangle$ of displacements before a diffusing atom (molecule, ion, particle), initiating its motion from a given site in the host lattice is irreversibly immobilized at a reaction site (trap, sink). The mean $\langle n\rangle$ is the first moment of the probability distribution function $\rho_{n}(t)$ which satisfies the underlying
Markovian stochastic master equation [Eq. (1)] and is related (in the infinite system limit) to the smallest eigenvalue of the corresponding evolution operator $(\mathbf{G})$. Except for the smallest lattices, the mean time $\langle t\rangle$ to absorption estimated using $\langle n\rangle$ is numerically indistinguishable from the exact value, rigorously defined as the reciprocal of the smallest eigenvalue in the spectrum characterizing the evolution of the system.

In calculating the mean walk length from the fundamental matrix of the theory, the matrix element $n(i, i)$ designates the number of times that a walker, starting from site $i$, revisits site $i$ in its passage through the lattice before being immobilized [see Ref. 22(b)]. The number of times the particle starting from site $i$ will transit through site $j$ before being trapped is the matrix element $n(i, j)$. The total number of times a diffusing particle starting at site $i$ will visit sites $i, j, k, \ldots$ on a lattice of $N$ sites,

$$
\langle n(i)\rangle=n(i, 1)+n(i, 2)+\cdots+n(i, i)+\cdots+n(i, N),
$$

is the site-specific walk length to the trap. If one sums the $\langle n(i)\rangle$ for the $i=1, \ldots, N-1$ nontrapping sites and divides by $N-1$, one obtains the overall mean walk length $\langle n\rangle$.

In the present problem, flows from environmental sites to a centrosymmetric trap are characterized by the overall, mean walk length $\langle n\rangle$. A RW from a central source $S$ to a target site on the boundary $B$ is characterized by the site-specific walk length $\langle n(S)\rangle$. Values of the mean walk length $\langle n\rangle$ (as well as higher-order moments) calculated using the theory of finite Markov processes are numerically exact; see Ref. [23].

\section{MARKOV THEORY: FLOWS FROM SATELLITE SITES TO A CENTROSYMMETRIC TRAP}

We consider first an atom or molecule undergoing equal a priori, nearest-neighbor displacements on a lattice before being immobilized (eventually) at a centrosymmetric reaction center. Documented in Ref. [23(b)] are numerically exact, sitespecific walk lengths $\langle n(i)\rangle$ for a RW on $d=2$, odd, periodic, square-planar lattices of $N=L \times L$ sites $(L=3, \ldots, 21)$ and, for each lattice, the overall mean walk length $\langle n\rangle$. Listed in Table A1 of the Supplemental Material [24] are the relevant results for the square-planar lattices considered in this article.

The fractal-like lattice [Fig. 1(b)] is a planar network with a distribution of sites inaccessible to a diffusing particle. Perhaps the simplest lattice that accounts for the presence of inaccessible sites is the Sierpinski "gasket," a deterministic fractal of fractal dimension $d_{f}=\ln 8 / \ln 3 \approx 1.8928$, but the centrosymmetric "site" of the gasket is a lacunary region, not a physical site. The fractal-like lattice introduced here has distributed, "inaccessible regions" but the centrosymmetric site is a "real" lattice site. By design, both the square-planar lattice and the fractal-like lattice have uniform coordination $(v=4)$ so, for fixed $N$, the site-specific walk lengths for both lattices can be compared and then studied as a function of system size. See Table I and Table A1 in the Supplemental Material [24].

For the problem as formulated, we can take advantage of classic results obtained by Montroll and Weiss [21] for random walks on regular, periodic lattices of uniform valence. These authors derived an asymptotic expression for the overall mean 
TABLE I. Diffusive flows from satellite sites to a centrosymmetric trap. The first line in each lattice entry gives the mean walk length for the regular, square-planar lattice [Fig. 1(a)] and the second line is the result for the fractal-like lattice [Fig. 1(b)]. Here, $S$ is the centrosymmetric site, $B(\mathrm{mp})$ a midpoint boundary site, and $B(c)$ a corner site. The quantity $\langle n(\mathrm{avg})\rangle$ is the overall mean walk length from all satellite sites and $\langle n(\operatorname{avg}[B])\rangle$ is the mean walk length from boundary sites only.

\begin{tabular}{lccccc}
\hline \hline$N=L \times L$ & $\langle n(S)\rangle$ & $\langle n[B(\mathrm{mp})]\rangle$ & $\langle n[B(c)]\rangle$ & $\langle n(\operatorname{avg})\rangle$ & $\langle n(\operatorname{avg}[B])\rangle$ \\
\hline $3 \times 3$ & 0 & 8 & 10 & 9 & 9 \\
$5 \times 5$ & 0 & 32 & 36 & 31.7 & 34 \\
& 0 & 40 & 48 & 38.3 & 45 \\
$7 \times 7$ & 0 & 74.5 & 81.1 & 71.6 & 77.7 \\
& 0 & 112 & 130 & 101 & 123.7 \\
$9 \times 9$ & 0 & 136.9 & 147.0 & 130.6 & 141.7 \\
& 0 & 240 & 272 & 209.8 & 261 \\
$11 \times 11$ & 0 & 220.56 & 235.0 & 209.9 & 227.5 \\
& 0 & 440 & 490 & 377.5 & 473 \\
$13 \times 13$ & 0 & 326.5 & 346.2 & 310.6 & 336.0 \\
& 0 & 728 & 800 & 617 & 775.7 \\
$15 \times 15$ & 0 & 455.5 & 481.4 & 433.5 & 468.1 \\
& 0 & 1120 & 1218 & 941 & 1185 \\
$17 \times 17$ & 0 & 637.1 & 639.3 & 579.4 & 624.5 \\
& 0 & 1632 & 1760 & 1362.3 & 1717 \\
\hline \hline
\end{tabular}

walk length to a centrosymmetric trap, viz.,

$$
\langle n\rangle=\left(\frac{N}{N-1}\right)\left[A_{1} N \ln N+A_{2} N+A_{3}+\frac{A_{4}}{N}\right],
$$

where, again, $N=L \times L$ is the total number of lattices sites and the $A_{i}$ are coefficients determined by the lattice symmetry. For the square-planar Euclidean lattice the coefficients are [21,25] $A_{1}=1 / \pi=0.318309886, A_{2}=0.195056166$, $A_{3}=-0.116964779$, and $A_{4}=0.484065704$. Notice that in the leading term the analytic dependence of $\langle n\rangle$ on the system size is transcendental. These authors also derived a fundamental invariance relation, viz., the mean number $\langle n\rangle$ of steps required for trapping from the site's nearest neighbor to a single deep trap,

$$
\langle n\rangle=N-1,
$$

a relation valid for all $d$-dimensional and fractal lattices of uniform valence. This relation provides a crucial check on the numerical accuracy of the results reported here, since the calculated value of $\langle n\rangle$ which satisfies Eq. (5) must be an integer. Inspection of the data reported in Table A1 (and Table A2) of the Supplemental Material [24] confirms that the Montroll invariance relation [Eq. (5)] is satisfied exactly in every case.

Before commenting on the quantitative difference in behavior described by the two data sets in Table A1 [24], note that, qualitatively, results for the regular square-planar lattice are integers or ratios of integers; the integer ratios rapidly become unwieldy, so after the first few lattices, the data recorded in Table A1 [24] for square-planar lattices are represented in decimal format. In contrast, the data for the fractal-like lattice are integers only. By analyzing the integer data for the site-specific walk lengths $\langle n(i)\rangle$ for a random walker on fractal-like lattices of increasing spatial extent, and using standard methods to sum sequences of integers (see Ref. [26]), we have derived a general, analytic expression for the $\langle n(i)\rangle$. We find

$$
\begin{aligned}
& \langle n(i)\rangle=4 \lambda \gamma(\gamma+1)-4 \sum_{i=1}^{\lambda} i(i-1)+2 \mu(2 \lambda-\mu), \quad \text { (6a) } \\
& \langle n(i)\rangle=4 \lambda \gamma(\gamma+1)-\left(\frac{4}{3}\right) \lambda(\lambda+1)(\lambda-1)+2 \mu(2 \lambda-\mu) .
\end{aligned}
$$

Here, $\lambda$ is the level index specifying the row of a given lattice of $N=L \times L$ sites with respect to the centrosymmetric site, $\gamma$ is the maximum value of the integer $\lambda$, and $\mu$ is the nearest-neighbor distance from site $i$ to the midpoint site of a given row [e.g., sites $1,3,6,10$, and 15 for the $N=11 \times 11$ lattice diagramed in Fig. 1(b)].

Using Eq. (6), an expression for the overall mean walk length $\langle n\rangle^{(\gamma)}$ corresponding to any generation $\gamma$ of the lattice having the distribution of grain boundaries diagramed in Fig. 1(b), follows at once. The resulting $\langle n\rangle^{(\gamma)}$ is the counterpart to Eq. (4) for square-planar lattices of uniform valence. In contrast to the asymptotic result derived by Montroll and Weiss for random walks on infinite periodic lattices with a centrosymmetric trap, the dependence of $\langle n(i)\rangle$ (and $\langle n\rangle^{(\gamma)}$ ) on the lattice size is algebraic, not transcendental. This characteristic of the fractal-like lattice [Fig. 1(b)] will also emerge in the following section when flows from satellite sites to one or a set of traps on the boundary of the lattice diagramed in Fig. 1(b) are considered.

Turning now to quantitative differences, summarized in Table I for the problem of diffusion from (all) satellite sites to a centrosymmetric trap are the data for the overall mean walk length as a function of the total number $N$ of lattice sites. Results for the regular, square-planar lattice and for the fractal-like lattice can be compared for each setting of $N$. Clearly, the influence of distributed grain boundaries compromises significantly the efficiency of the underlying diffusion-reaction process, a difference which becomes more pronounced with increasing lattice size.

It is also instructive to study diffusion to a centrosymmetric trap from boundary sites only. For example, for the $N=$ $11 \times 11$ lattices displayed in Fig. 1, the difference in values of $\langle n\rangle$ for the square-planar versus the fractal-like lattice is a factor of 1.8 when all satellite sites are considered. When only sites farthest removed from the trap (i.e., boundary sites) are taken into account, the difference is a factor $\approx 15 \%$ larger.

\section{MARKOV THEORY: FLOWS FROM SATELLITE SITES TO A BOUNDARY TRAP}

We turn now to the "inverse" problem, viz., characterizing the history of a particle emitted from a centrosymmetric "source" site $S$, subsequently undergoing random displacements on the lattice, and immobilized, eventually, at one or more target sites on the boundary. In Sec. III, advantage was taken of the fact that, in specifying the position of the trap, the centrosymmetric site on an odd $N=L \times L$ lattice is unique. In the "inverse" problem, there are $4(L-1)$ boundary sites at 
(a)

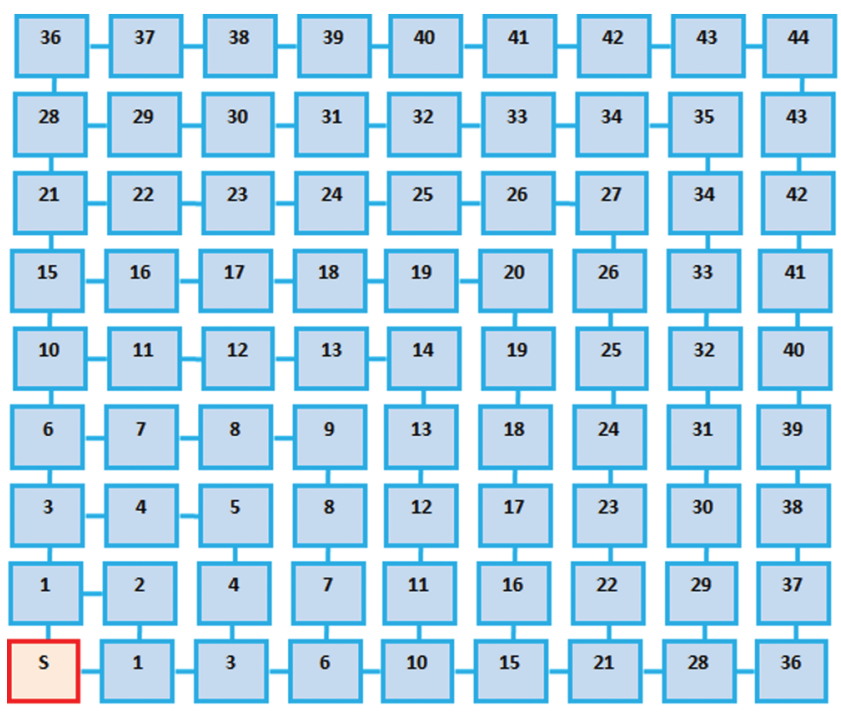

(c)

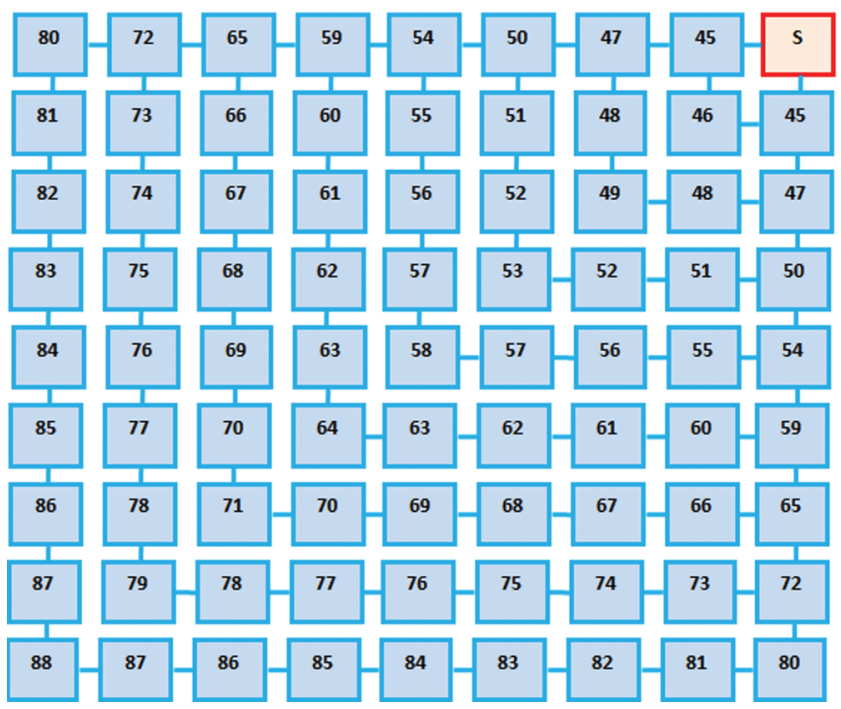

(b)

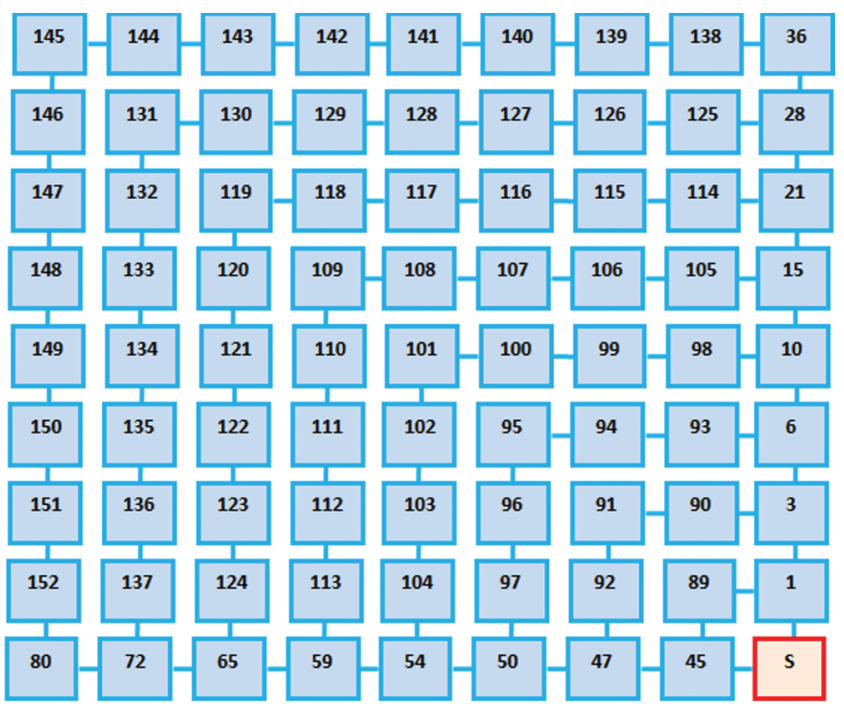

(d)

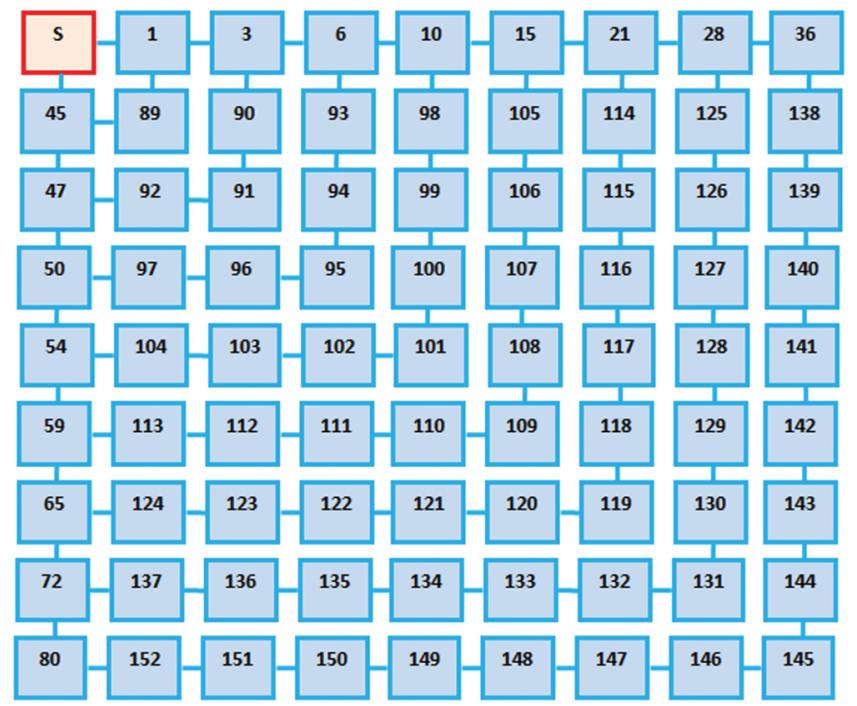

FIG. 2. (Color online) Symmetry-equivalent sites on a $17 \times 17$ fractal-like lattice with a sink at the upper-right-hand corner site. The companion regular, square-planar lattice has the same site specifications. Shown are (a) the upper-right-hand quadrant (I), (b) the upper-left-hand quadrant (II), (c) the lower-left-hand quadrant (III), and (d) the lower-right-hand quadrant (IV). The corner trap locations are site 2 on a $3 \times 3$ lattice, site 5 on a $5 \times 5$ lattice, site 9 on a $7 \times 7$ lattice, site 14 on a $9 \times 9$ lattice, site 20 on a $11 \times 11$ lattice, site 27 on a $13 \times 13$ lattice, site 35 on a $15 \times 15$ lattice, and site 44 on the $17 \times 17$ lattice displayed in this figure.

which one can position a trap. In this study, we first place a trap at the lattice site farthest removed from the centrosymmetic one, viz., at the upper-right-hand corner of the regular or fractal-like lattice. We calculate the mean walk length $\langle n(S)\rangle$ to that site of a particle initiating its motion at (emitted from) a centrosymmetric source $S$. Analytic results will also be presented for random walks initiated at the nearest boundary midpoint site $B(\mathrm{mp})$ to the $\operatorname{trap}\langle n[B(\mathrm{mp})]\rangle$ and at the nearest corner site $B(c)$ to the trap $\langle n[B(c)]\rangle$.

A lattice with a corner trap can be parsed into symmetrydistinct quadrants: upper right (I), upper left (II), lower left (III), and lower right (IV) (see Fig. 2). The sites in the upperright-hand quadrant (I) are labeled as in Fig. 1(b). Sites in the upper-left-hand quadrant (II) and lower-right-hand quadrant
(IV) are symmetry related; the long diagonal from the upper right to the lower left is a $C_{2}$ symmetry axis. Sites in the lower-left-hand quadrant (III) are also symmetry related with respect to this $C_{2}$ axis.

As noted previously, accuracy of the numerical results obtained can be checked by using Eq. (5). For example, on the $17 \times 17$ fractal-like lattice, sites 43 are nearest-neighbor sites to the trap at site 44 (see Fig. 2); we calculate $\langle n(43)\rangle=576$. Virtual transitions to "off lattice" nearest-neighbor sites are set to zero. Then $[576+576+0+0] / 4=288=N-1$.

The calculated site-specific walk lengths for a RW on a square-planar lattice with a corner trap are integers or ratios of integers. As for the case of a centrosymmetric trap (see the previous section), these ratios rapidly become unwieldy, so 
TABLE II. Diffusive flows from satellite sites to boundary traps. The first line in each lattice entry gives the result for the regular, square-planar lattice and the second line for the fractal-like lattice (Fig. 2). The entry $\left\langle n\left(S_{b}\right)\right\rangle$ gives the mean walk length from the centrosymmetric source $S$ to the boundary, where all boundary sites are deep traps.

\begin{tabular}{lrcrrc}
\hline \hline$N=L \times L$ & $\langle n(S)\rangle$ & $\langle n[B(\mathrm{mp})]\rangle$ & $\langle n[B(c)]\rangle$ & $\langle n(\mathrm{avg})\rangle$ & $\left\langle n\left(S_{b}\right)\right\rangle$ \\
\hline $3 \times 3$ & 21.5 & 16 & 22.5 & 21.9 & 1 \\
$5 \times 5$ & 90.7 & 74.1 & 95.1 & 88.7 & 4.5 \\
& 123.4 & 92 & 121.4 & 116.9 & 10 \\
$7 \times 7$ & 218.9 & 186.0 & 228.5 & 212.4 & 10.4 \\
& 367.2 & 276 & 350.2 & 343.1 & 35 \\
$9 \times 9$ & 413.1 & 358.6 & 429.7 & 400.1 & 18.6 \\
& 813.2 & 616 & 761.2 & 757.7 & 84 \\
$11 \times 11$ & 678.5 & 597.0 & 703.9 & 657.1 & 29.2 \\
& 1521.1 & 1160 & 1406.1 & 1417.2 & 165 \\
$13 \times 13$ & 1019.1 & 905.4 & 1055.1 & 981.7 & 42.2 \\
& 2550.2 & 1956 & 2336.2 & 2377.6 & 286 \\
$15 \times 15$ & 1438.5 & 1287.2 & 1486.8 & 1379.8 & 57.5 \\
& 3959.7 & 3052 & 3602.7 & 3694.9 & 455 \\
$17 \times 17$ & 1939.5 & 1745.2 & 2002.0 & 1865.5 & 75.2 \\
& 5808.4 & 4496 & 5256.4 & 5424.8 & 680 \\
\hline \hline
\end{tabular}

the data for the square-planar lattice are presented in decimal format (Table II and Table A2 in the Supplemental Material [24]). For the fractal-like lattice, the site-specific walk lengths are also integers or ratios of integers, and these, as well, become more and more cumbersome with increase in lattice size (Table A2 [24]). However, it is only by tabulating the data in integer format that one notices unexpected, and rather surprising, integer relationships between the site-specific walk lengths for certain pairs of sites. We will show below that these relationships can be used to determine analytic solutions to the RW problem in two specific cases.

A random walker emitted from a centrosymmetric source $S$ will transit through the lattice, visiting other sites (cycling back to revisit site $S$ is not excluded) before hitting the (deep) trap and being immobilized there irreversibly; for the $17 \times 17$ fractal-like lattice, the average number of displacements is equal to $\langle n(S)\rangle=8374133440 / 1441729 \approx 5808.4$.

Random walks initiated at either of the two boundary midpoint sites $B(\mathrm{mp})$ closest to the corner trap site on the $17 \times 17$ fractal-like lattice (viz., sites 36 ) can also be calculated. Once initialized at the site $B(\mathrm{mp})$, a random walker will, with nonzero probability, subsequently visit all nontrapping sites of the lattice [including $B(\mathrm{mp})$ ] before the random walk terminates at the target site; on the $17 \times 17$ fractal-like lattice, calculation gives $\langle n[B(\mathrm{mp})]\rangle=\langle n(36)\rangle=4496$, which is an integer. Examining the complete body of data on fractal-like lattices (Table A2 [24]), one finds that all the $\langle n[B(\mathrm{mp})]\rangle$ are integers, and the methods described in Ref. [26] for analyzing sequences of integers can again be invoked, yielding the following exact, analytic result for $\langle n[B(\mathrm{mp})]\rangle$ :

$$
\begin{aligned}
\langle n[B(\mathrm{mp})]\rangle= & 16+76(m-1)+54(m-1)(m-2) \\
& +8(m-1)(m-2)(m-3)
\end{aligned}
$$

where

$$
m=(L-1) / 2 \text {, }
$$

which reduces to

$$
\langle n[B(\mathrm{mp})]\rangle=(L-1)\left[L^{2}-\left(\frac{1}{2}\right)(L-1)\right] .
$$

We draw attention to the algebraic dependence of $\langle n[B(\mathrm{mp})]\rangle$ on $L$, noting that in the large-system limit $\langle n[B(\mathrm{mp})]\rangle$ behaves as $L^{3}$, that is, of order $O(N \sqrt{ } N)$.

For a deep trap positioned at one corner of the fractal-like lattice, we denote by $B(c)$ either of the two corner boundary sites closest to the trap; on the $17 \times 17$ fractal-like lattice, the two symmetry-equivalent sites are labeled 145. In this case, one finds (see Table A2 [24]) $\langle n(S)\rangle-\langle n(145)\rangle=8374133440 /$ $1441729-7578299032 / 1441729=552$. The difference is an integer, a remarkable result, since the only prime common to the numerators is 2 , and the denominator, 1441729 , is a prime number. On analyzing the full data set for the fractal-like lattices considered here $(3 \times 3$ to $17 \times 17)$, one finds that all the differences, $\langle n(S)\rangle-\langle n[B(c)]\rangle$, are integers and the methods of Ref. [26] lead to the following exact, analytic result:

$$
\begin{aligned}
& \langle n(S)\rangle-\langle n[B(c)]\rangle=\sum_{i=0}^{\gamma}\left(2 i^{2}-1\right), \\
& \langle n(S)\rangle-\langle n[B[c]]\rangle=\left(\frac{2}{3}\right) \gamma(\gamma-1)(2 \gamma-1)-\gamma,
\end{aligned}
$$

where the generation index $\gamma=(L-1) / 2$. Once again, the functional dependence is algebraic not transcendental and, as for the limiting behavior of $\langle n[B(\mathrm{mp})]\rangle$ [Eq. (8)], for a large-system size, the right-hand side of Eq. (9) increases as $L^{3}$, that is, of order $O(N \sqrt{ } N)$.

Less surprising, but also of interest, is the difference in site-specific walk lengths between adjacent sites on the boundary of each fractal-like lattice. Owing to the symmetry breaking introduced by displacing the target site from the centrosymmetric site to a corner site, results here are best presented sequentially for each quadrant (see Fig. 2).

To illustrate, for the $11 \times 11$ fractal-like lattice, the deep trap is positioned at the (corner) site (labeled 20) in quadrant I. From the Montroll-Weiss invariance relation [Eq. (5)], the difference in mean walk lengths between the trap and one of its nearest-neighbor sites is $n(19)-n(T)=240$. Inspection of the data in Table A2 [24] shows $n(18)-n(19)=236$, $n(17)-n(18)=232, n(16)-n(17)=228$, and $n(15)-n(16)$ $=224$. The site-specific walk lengths are integers, and the differences are, uniformly, equal to 4 .

Less obvious is that the differences between adjacent sites on the boundary of quadrant III also reduce to integers with a uniform separation of 4 :

$$
\begin{aligned}
& n(63)-n(62)=1236889 / 773-1232251 / 773=6 \\
& n(62)-n(61)=1232251 / 773-1224521 / 773=10 \\
& n(61)-n(60)=1224521 / 773-1213699 / 773=14 \\
& n(60)-n(59)=1213699 / 773-1199785 / 773=18
\end{aligned}
$$


Given the design of the fractal-like lattice, and the options available to a RW on the boundary (two possible displacements to a nearest-neighbor site, and two virtual transitions), the uniform difference of 4 necessarily follows. In quadrant III, the difference in mean walk length between the corner site 64 on the $11 \times 11$ fractal-like lattice and either of the two symmetry-equivalent sites (63) immediately adjacent to site 64 is

$$
n(64)-n(63)=1238435 / 773-1236889 / 773=2 .
$$

A similar result is found for the corner site in quadrant III for all the fractal-like lattices. This result may be compared with the one derived for odd, periodic, square-planar lattices with a centrosymmetric trap, where it can be proved this integer difference of 2 is valid for any corner site [see Ref. 23(b)]. More generally, this result is valid for any corner site on a lattice of uniform coordination $v=4$.

Apart from the corner sites, differences between adjacent border sites in quadrants II and IV are separated, uniformly, by the integer 4 plus a constant "shift" $\Delta$. The relation " $4+\Delta$ " holds for all fractal-like lattices of the design introduced here, but the "shift" $\Delta$ turns out to be lattice specific, with a different (constant) value for each lattice; in the present example, $\Delta=327 / 1546$. We have not, as yet, been able to derive a general expression that predicts the value of $\Delta$ for each fractal-like lattice; were that possible, a general analytic solution to the RW problem for a single corner trap could be written down at once and compared with the analytic result reported in the previous section for ergodic flows to a single, centrosymmetric trap. More generally, it is probable that the difference in values of the "shift" $\Delta$ (in quadrants I and III, $\Delta=0$; in quadrants II and IV, $\Delta \neq 0$ ) is linked to the somewhat different algebraic structure of Eqs. (9) and (8); in the large-system limit, for example, Eq. (9) behaves as (2/3) $N \sqrt{ } N$ whereas Eq. (8) behaves as $N \sqrt{ } N$.

Notice that the design of the fractal-like geometry ensures that a random walker proceeding, eventually, to the corner trap from any site of the lattice must pass through one of the boundary midpoint sites $B(\mathrm{mp})$ closest to the corner trap. Assuming a characteristic "jump time," a lower bound on the time required for a particle to encounter the "gateway" site on the boundary $B(\mathrm{mp})$ would be a "straight line" trajectory from $S$ to $B(\mathrm{mp})$. The difference in walk lengths,

$$
\langle n(S)\rangle-\langle n[B(\mathrm{mp})]\rangle=1312.4,
$$

or, more generally, the difference $\langle n(S)\rangle-\langle n[B(\mathrm{mp})]\rangle$, with $\langle n[B(\mathrm{mp})]\rangle$ given by the analytic expression Eq. (8), gives one estimate of the time required for a particle emitted at the source $S$ to wander through the $17 \times 17$ fractal-like lattice before encountering a midpoint boundary site nearest the trap.

Similarly, the difference $\langle n(S)\rangle-\langle n[B(C)]\rangle$, with $\langle n[B(C)]\rangle$ given by Eq. (9), translates into an estimate of the time required for a particle emitted from site $S$ on an $L \times L$ fractal-like lattice, visiting subsequently with nonzero probability all nontrapping sites of the lattice (including site $S$ ), to reach either of the two corner sites nearest the trap.

In this section, we considered first the case of a single corner trap to provide a comparison with results obtained in the classical RW problem analyzed in the previous section, viz., a single trap at the centrosymmetric site of the lattice. It is also of interest to study the lattice statistical counterpart to an absorbing boundary; this can be done by placing a deep trap at all $4(L-1)$ boundary sites of a given $N=L \times L$ lattice.

We discuss first the case where a particle emitted from a centrosymmetric source undergoes random displacements, visiting all possible lattice sites on the fractal-like lattice, and then is trapped irreversibly upon reaching any site on the boundary. Values of $\left\langle n\left(S_{b}\right)\right\rangle$ for this case are listed in Table II, and analysis of the results (using the methods noted previously) leads to the following, closed-form, analytic expression:

$$
\left\langle n\left(S_{b}\right)\right\rangle=(1 / 6)(L-1)\left[(L-1)^{2}-1\right] .
$$

In the limit of large-system size, $\left\langle n\left(S_{b}\right)\right\rangle$ behaves as $\left\langle n\left(S_{b}\right)\right\rangle \sim N \sqrt{ } N$. By contrast, a plot of the companion data in Table II for the square-planar Euclidean lattice shows a strictly linear dependence of $\left\langle n\left(S_{b}\right)\right\rangle$ on $N$ (with $R^{2}=0.9995$ ), thus providing further (analytic) evidence that breaking the symmetry of the host lattice by introducing inhomogeneities leads to a deterioration in reaction efficiency.

\section{CONTINUUM THEORY: FLOWS FROM ENVIRONMENTAL SITES TO A CENTROSYMMETRIC TRAP}

We now take up sequentially the two problems described in Secs. III and IV, and derive, using classical diffusion theory (see Ref. [27]), analytic results to complement those obtained previously for a regular, square-planar lattice. In this section we consider the continuum analog of the lattice problem discussed in Sec. III, whereas the continuum counterpart of the problem addressed in Sec. IV is dealt with in the next section.

Consider first a square with reflecting sides of length $\Lambda$ and a small interior square with absorbing sides of length $\ell$ (see Fig. 3). We wish to determine the mean absorption time $\langle t\rangle$ for a particle that starts off from a given position $\left(x_{0}, y_{0}\right)$ in the region between the boundaries of both squares.

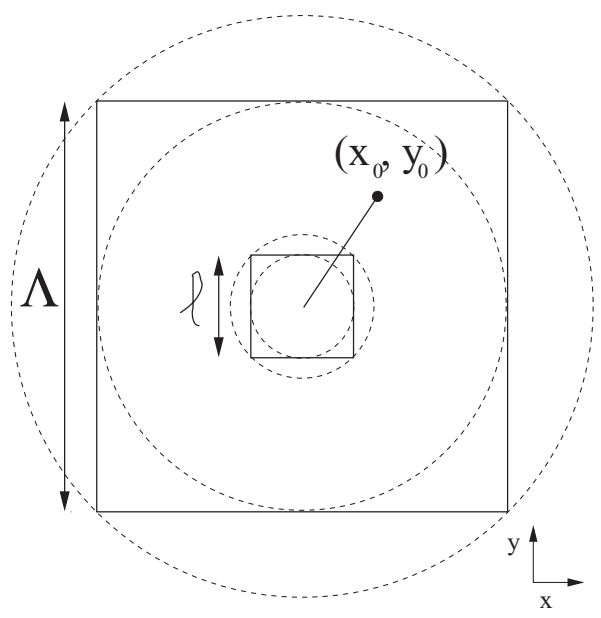

FIG. 3. Set of two concentric squares of respective side lengths $\Lambda$ and $\ell$. The boundary of the outer square is assumed to be reflecting, whereas the boundary of the inner square is assumed to be fully absorbing. The respective incircles and excircles used for the construction of a lower bound and an upper bound to the exact solution for the absorption time $\langle t\rangle$ of a Brownian particle starting from a hypothetical initial position $\left(x_{0}, y_{0}\right)$ are shown in dashed lines. 
The solution of this problem in Cartesian coordinates can be obtained by the method of images, but the calculations are fairly cumbersome. Since we are mainly interested in the system size dependence of the solution, a quick estimate for the behavior of $\langle t\rangle$ can be obtained by considering a spherically symmetric problem: the computation of $\langle t\rangle_{r_{0}}$ for a particle that starts diffusing at a distance $r_{0}$ from the common center of a small, fully absorbing circle of radius $r<r_{0}$ and a large circle of radius $R>r_{0}$ with a reflecting boundary (see Fig. 3 ). This problem can be solved via Laplace transform methods; see Ref. [27(b)] for details (Secs. 6.4.2 and 6.4.3). There, an explicit expression for $\tilde{F}(u) \equiv \int_{0}^{\infty} d t e^{-u t} F(t)$, defined as the Laplace transform of the (negative) time derivative of the absorption probability density $F(t)=-\frac{d S}{d t}$, can be obtained, viz.,

$$
\tilde{F}(u)=\frac{I_{0}\left(\sqrt{\frac{u}{D}} r_{0}\right) K_{-1}\left(\sqrt{\frac{u}{D}} R\right)+K_{0}\left(\sqrt{\frac{u}{D}} r_{0}\right) I_{-1}\left(\sqrt{\frac{u}{D}} R\right)}{I_{0}\left(\sqrt{\frac{u}{D}} r\right) K_{-1}\left(\sqrt{\frac{u}{D}} R\right)+K_{0}\left(\sqrt{\frac{u}{D}} r\right) I_{-1}\left(\sqrt{\frac{u}{D}} R\right)} .
$$

Here, $I_{n}(\cdot)$ and $K_{n}(\cdot)$ are, respectively, modified Bessel functions of the first and second kind. From the above expression, it is possible to extract an exact expression for $\langle t\rangle$.

The Laplace transform of the probability density is related to the Laplace transform of the survival probability as follows:

$$
\tilde{F}(u)=S(0)-u \tilde{S}(u)=1-u \tilde{S}(u) .
$$

That is,

$$
\tilde{S}(u)=\frac{1-\tilde{F}(u)}{u} .
$$

One also has

$$
\langle t\rangle_{r_{0}}=\int_{0}^{\infty} d t t F(t)=-\left.t S(t)\right|_{0} ^{\infty}+\int_{0}^{\infty} d t S(t) .
$$

In the present case, $S(t)$ vanishes faster than $t^{-1}$ as $t \rightarrow \infty$; therefore,

$$
\langle t\rangle_{r_{0}}=\int_{0}^{\infty} d t S(t)=\lim _{u \rightarrow 0} \tilde{S}(u)=\lim _{u \rightarrow 0} \frac{1-\tilde{F}(u)}{u} .
$$

Using the small- $u$ expansion for the Bessel functions in the expression for $\tilde{F}(u)$, we get

$$
\langle t\rangle_{r_{0}}=\frac{1}{4 D}\left[2 R^{2} \ln \left(\frac{r_{0}}{r}\right)-\left(r_{0}^{2}-r^{2}\right)\right] .
$$

Note that $\langle t\rangle \rightarrow 0$ as $r_{0} \rightarrow r$, as should be the case. Note also that the absorption time diverges logarithmically as we let the inner circle shrink to a point $(r \rightarrow 0)$ (see Fig. 3).

Returning to the original problem, a domain with two concentric squares, in order to obtain a lower and an upper bound for the absorption time we assume that the particle is initially at a location $\left(x_{0}, y_{0}\right)$ with $r_{0}=\sqrt{x_{0}^{2}+y_{0}^{2}}$. To get a lower bound $\langle t\rangle^{L}$ for $\langle t\rangle$, we take the incircle of the outer square and the excircle of the inner square, that is, we take $R=\Lambda / 2$ and $r=\ell / \sqrt{2}$. The result is

$$
\langle t\rangle^{L}=\frac{1}{4 D}\left[\frac{\Lambda^{2}}{2} \ln \left(\frac{\sqrt{2\left(x_{0}^{2}+y_{0}^{2}\right)}}{\ell}\right)-\left(x_{0}^{2}+y_{0}^{2}-\frac{\ell^{2}}{2}\right)\right] .
$$

To obtain an upper bound, we need to take the excircle of the outer square and the incircle of the inner square, i.e., $R=\Lambda / \sqrt{2}$ and $r=\ell / 2$. We find

$\langle t\rangle^{U}=\frac{1}{4 D}\left[\Lambda^{2} \ln \left(\frac{2 \sqrt{\left(x_{0}^{2}+y_{0}^{2}\right)}}{\ell}\right)-\left(x_{0}^{2}+y_{0}^{2}-\frac{\ell^{2}}{4}\right)\right]$.

For large $\Lambda$, the term proportional to $\Lambda^{2}$ becomes dominant, both for the upper bound and for the lower bound. Thus, up to a multiplicative constant, the absorption time $\langle t\rangle^{L}<\langle t\rangle\left\langle\langle t\rangle^{U}\right.$ behaves as

$$
\langle t\rangle \propto \Lambda^{2} \ln \left[\sqrt{\left(x_{0}^{2}+y_{0}^{2}\right)}\right],
$$

where we have assumed that $\sqrt{\left(x_{0}^{2}+y_{0}^{2}\right)}$ is sufficiently large, so that the remaining terms in the expression for $\langle t\rangle^{U}$ and $\langle t\rangle^{L}$ become subdominant in the joint limit $\Lambda, r_{0} \rightarrow \infty$. This is in agreement with the result obtained by Montroll [see Ref. 21(b)] for the site-specific walk length on a $d=2$ dimensional regular $N$-site lattice, namely,

$$
\langle n\rangle(j) \propto N \ln |j|,
$$

where $j$ is the coordinate vector of the walker's initial position measured from the absorbing site, which is taken to be the origin of the coordinate system.

Finally, we construct an upper and a lower bound for $\langle t\rangle_{\text {uniform, }}$, i.e., the mean absorption time for a homogeneous initial condition (global absorption time). Here, we average $\langle t\rangle_{r_{0}}$ over all the volume elements of the region between the two circles:

$$
\begin{aligned}
\langle t\rangle_{\text {uniform }}^{\text {circle }} & =\frac{1}{\pi\left(R^{2}-r^{2}\right)} \int_{r}^{R} 2 \pi\langle t\rangle_{r_{0}} r_{0} d r_{0} \\
& =\frac{1}{4 D}\left(\frac{2 R^{4}}{R^{2}-r^{2}} \ln \frac{R}{r}-\frac{3}{2} R^{2}+\frac{r^{2}}{2}\right)
\end{aligned}
$$

(one can easily check as a consistency test that the limit of the above expression as $r \rightarrow R$ goes to zero). Proceeding as in the case of a delta initial condition, we find the following lower and upper bounds for the absorption time in a double square domain. The lower bound reads

$$
\begin{aligned}
\langle t\rangle_{\text {uniform }}^{L} & =\left.\langle t\rangle_{\text {uniform }}^{\text {circle }}\right|_{r=\ell / \sqrt{2}, R=\Lambda / 2} \\
& =\frac{1}{8 D}\left(\frac{\Lambda^{4}}{\Lambda^{2}-2 \ell^{2}} \ln \frac{\Lambda}{\sqrt{2} \ell}-\frac{3}{4} \Lambda^{2}+\frac{\ell^{2}}{2}\right),
\end{aligned}
$$

whereas the upper bound reads

$$
\begin{aligned}
\langle t\rangle_{\text {uniform }}^{U} & =\left.\langle t\rangle_{\text {uniform }}^{\text {circle }}\right|_{r=\ell / 2, R=\Lambda / \sqrt{2}} \\
& =\frac{1}{8 D}\left(\frac{4 \Lambda^{4}}{2 \Lambda^{2}-\ell^{2}} \ln \frac{\sqrt{2} \Lambda}{\ell}-\frac{3}{2} \Lambda^{2}+\frac{\ell^{2}}{4}\right)
\end{aligned}
$$

We conclude that, up to a multiplicative constant for which bounds can be obtained, for large $L$ the absorption time $\langle t\rangle^{L}<$ $\langle t\rangle\left\langle\langle t\rangle^{U}\right.$ behaves as $\langle t\rangle \propto \Lambda^{2} \ln \left(\Lambda^{2}\right)$, in agreement with the $N \ln N$ behavior established in Ref. [21(b)] in the large $N$ limit 
for the mean walk length on a $d=2$ dimensional Euclidean lattice [cf. Eq. (4)].

\section{CONTINUUM THEORY: FLOWS FROM A CENTROSYMMETRIC SOURCE TO THE BOUNDARY}

To develop a continuum theory to complement the lattice statistical one elaborated on in Sec. IV, we need to compute the absorption time of a diffusing point particle enclosed in a square with a fully absorbing boundary of side length $\Lambda$ (we assume that the origin of our coordinate system is placed at the left bottom corner of the square). In other words, we need to solve the following boundary value problem for the distribution function ("concentration") $P(x, y, t)$ :

$$
\begin{aligned}
\frac{\partial P}{\partial t} & =D\left[\frac{\partial^{2} P}{\partial x^{2}}+\frac{\partial^{2} P}{\partial y^{2}}\right], \\
P(x=0, y, t) & =P(x=\Lambda, y, t)=0, \\
P(x, y=0, t) & =P(x, y=\Lambda, t)=0 .
\end{aligned}
$$

We shall obtain the solution for a general initial condition $P(x, y, t=0) \equiv P_{0}(x, y)$ and subsequently consider specific cases, e.g., the case of a point source located at some point $\left(x_{0}, y_{0}\right)$ and the case of a uniform initial distribution $P_{0}(x, y)=$ $\frac{1}{\Lambda^{2}}$.

The solution for the above problem can be obtained by the method of separation of variables [27]. To this end, we make the ansatz $P(x, y, t)=X(x) Y(y) T(t)$ and solve the corresponding eigenvalue problems for each of the functions $X(x), Y(y)$, and $T(t)$. This leads to the following expression:

$$
\begin{aligned}
P(x, y, t)= & \sum_{n_{x}=1}^{\infty} \sum_{n_{y}=1}^{\infty} A_{n_{x} n_{y}} \sin \left(n_{x} \frac{\pi}{\Lambda} x\right) \sin \left(n_{y} \frac{\pi}{\Lambda} y\right) \\
& \times \exp \left(-D\left(n_{x}^{2}+n_{y}^{2}\right) \frac{\pi^{2}}{\Lambda^{2}} t\right) .
\end{aligned}
$$

From the form of this solution it is clear that for sufficiently long times $t \gg \frac{\Lambda^{2}}{D}$ the exponential decay of the solution will be governed by the smallest eigenvalue of the temporal part $T(t)$. Thus, the solution (which is no longer normalized for $t>0$ ) reduces to a single, exponentially decaying mode:

$$
P(x, y, t) \approx A_{11} \sin \left(\frac{\pi}{\Lambda} x\right) \sin \left(\frac{\pi}{\Lambda} y\right) \exp \left(-2 D \frac{\pi^{2}}{\Lambda^{2}} t\right) .
$$

This is in contrast with the $t^{-1}$ decay displayed by the propagator (free diffusion).

In the long-time limit the boundaries significantly modify the time behavior of the solution. The coefficients $A_{n_{x} n_{y}}$ are related to the initial condition and can be obtained as follows. Using the fact that the sine functions constitute a set of orthonormal eigenfunctions, viz.,

$$
\int_{0}^{L} d u \sin \left(n \frac{\pi}{\Lambda} u\right) \sin \left(m \frac{\pi}{\Lambda} u\right)=\frac{\Lambda}{2} \delta_{n m}, n, m=1,2, \ldots,
$$

in the generic expression for the initial condition

$$
P_{0}(x, y)=\sum_{n_{x}=1}^{\infty} \sum_{n_{y}=1}^{\infty} A_{n_{x} n_{y}} \sin \left(n_{x} \frac{\pi}{\Lambda} x\right) \sin \left(n_{y} \frac{\pi}{\Lambda} y\right),
$$

we obtain a general expression for the coefficients $A_{n_{x} n_{y}}$ in terms of the initial condition, viz.,

$$
A_{n_{x} n_{y}}=\frac{4}{\Lambda^{2}} \int_{0}^{L} \int_{0}^{L} d x d y P_{0}(x, y) \sin \left(n_{x} \frac{\pi}{\Lambda} x\right) \sin \left(n_{y} \frac{\pi}{\Lambda} y\right) \text {. }
$$

We now evaluate these coefficients for specific initial conditions. Assuming that a point particle is initially released at a given interior point $\left(x_{0}, y_{0}\right)$ with $0<x_{0}, y_{0}<\Lambda$, i.e.,

$$
P_{0}(x, y)=\delta\left(x-x_{0}\right) \delta\left(y-y_{0}\right)
$$

one obtains

$$
A_{n_{x} n_{y}}=\left(\frac{4}{\Lambda^{2}}\right) \sin \left(n_{x} \frac{\pi}{\Lambda} x_{0}\right) \sin \left(n_{y} \frac{\pi}{\Lambda} y_{0}\right) .
$$

In particular, when the source is initially at the center of the square $x_{0}=y_{0}=\frac{\Lambda}{2}$, then

$$
A_{n_{x} n_{y}}=\left\{\begin{array}{cc}
\left(\frac{4}{\Lambda^{2}}\right) \sin \left(n_{x} \frac{\pi}{2}\right) \sin \left(n_{y} \frac{\pi}{2}\right)=(-1)^{\frac{\left(n_{x}+n_{y}\right)}{2}+1}\left(\frac{4}{\Lambda^{2}}\right), & \text { if both } n_{x}, n_{y} \text { odd } \\
0, & \text { otherwise }
\end{array}\right.
$$

If the diffusing particle is initially located with equal probability anywhere inside the square (homogeneous initial state), one finds

$$
A_{n_{x} n_{y}}=\left\{\begin{array}{cc}
\frac{16}{\pi^{2} L^{2} n_{x} n_{y}}, & \text { if both } n_{x}, n_{y} \text { odd } \\
0, & \text { otherwise }
\end{array}\right.
$$

[This result could also have been obtained by taking a uniform average the result for a specific $\left(x_{0}, y_{0}\right)$ over all possible values of $x_{0}$ and $y_{0}$.]
In order to obtain the survival probability of the particle $S(t)$ up to time $t$ we must take the spatial integral of the distribution $P(x, y, t)$ over the region with nonzero particle concentration [see Ref. 22(b)],

$$
S(t)=\int_{0}^{L} \int_{0}^{L} d x d y P(x, y, t) .
$$

This leads to the following expression,

$$
S(t)=\sum_{n_{x}=1}^{\infty} \sum_{n_{y}=1}^{\infty} B_{n_{x} n_{y}} \exp \left(-D\left(n_{x}^{2}+n_{y}^{2}\right) \frac{\pi^{2}}{\Lambda^{2}} t\right)
$$


with $B_{n_{x} n_{y}}=\frac{4 \Lambda^{2}}{\pi^{2} n_{x} n_{y}} A_{n_{x} n_{y}}$. Both for a delta-peaked initial condition and for a uniform initial condition the sums run only over odd values of $n_{x}, n_{y}$. The mean trapping time $\langle t\rangle$ at the boundaries (which is the analog of the mean walk length multiplied by the time scale of a single hopping event in the lattice problem) is given by the formula [see Ref. 22(b)]

$$
\langle t\rangle=-\int_{0}^{\infty} d t t \frac{d S}{d t},
$$

which, in the present problem, takes the form

$$
\langle t\rangle=\frac{\Lambda^{2}}{\pi^{2} D} \sum_{n_{x}=1}^{\infty} \sum_{n_{y}=1}^{\infty} \frac{B_{n_{x} n_{y}}}{\left(n_{x}^{2}+n_{y}^{2}\right)} .
$$

The terms of the series are expected to decrease rapidly as the odd-valued $n_{x}$ and $n_{y}$ become large, hence we make the approximation

$$
\langle t\rangle \approx \frac{B_{11}}{2} \frac{\Lambda^{2}}{\pi^{2} D}=\frac{2 \Lambda^{4}}{\pi^{4} D} A_{11},
$$

which becomes

$$
\langle t\rangle \approx \frac{8 \Lambda^{2}}{\pi^{4} D} \sin \left(\frac{\pi}{\Lambda} x_{0}\right) \sin \left(\frac{\pi}{\Lambda} y_{0}\right) .
$$

(In particular, one has $\langle t\rangle_{\text {center }} \approx \frac{8 \Lambda^{2}}{\pi^{4} D}=0.08213 \frac{\Lambda^{2}}{D}$ when the particle starts off at the center of the square, where the trapping time reaches its maximum as a function of $x_{0}$ and $y_{0}$.)

However, if the initial condition is homogeneous, one obtains the (smaller) value

$$
\langle t\rangle_{\text {uniform }} \approx \frac{32 \Lambda^{2}}{\pi^{6} D}=0.03328 \frac{\Lambda^{2}}{D} .
$$

Albeit fairly accurate, these results are slightly different from the exact ones. The exact results are obtained upon numerical evaluation of the corresponding double series, and read as follows:

$$
\begin{aligned}
\langle t\rangle_{\text {center }} & =\frac{16 \Lambda^{2}}{\pi^{4} D} \sum_{\substack{n_{x}=1 \\
n_{x} \text { odd }}}^{\infty} \sum_{\substack{n_{y}=1 \\
n_{y} \text { odd }}}^{\infty} \frac{(-1)^{\left(n_{x}+n_{y}\right) / 2+1}}{n_{x} n_{y}\left(n_{x}^{2}+n_{y}^{2}\right)} \\
& =\frac{16 \times 0.4485}{\pi^{4}} \frac{\Lambda^{2}}{D}=0.07367 \frac{\Lambda^{2}}{D}
\end{aligned}
$$

and

$$
\begin{aligned}
\langle t\rangle_{\text {uniform }} & =\frac{64 \Lambda^{2}}{\pi^{6} D} \sum_{\substack{n_{x}=1 \\
n_{x} \text { odd }}}^{\infty} \sum_{\substack{n_{y}=1 \\
n_{y} \text { odd }}}^{\infty} \frac{1}{n_{x}^{2} n_{y}^{2}\left(n_{x}^{2}+n_{y}^{2}\right)} \\
& =\frac{64 \times 0.5279}{\pi^{6}} \frac{\Lambda^{2}}{D}=0.03514 \frac{\Lambda^{2}}{D} .
\end{aligned}
$$

In order to compare the results for $\langle t\rangle_{\text {center }}$ and $\langle t\rangle_{\text {uniform }}$ with the site-specific and the global mean walk length for the square lattice problem, we need to take into account that the typical time scale for nearest-neighbor hopping for the random walk model is $\Delta t=(\Delta \ell)^{2} /(4 D)$, where the lattice constant is $\Delta \ell \approx \frac{\Lambda}{L-1}$ and $\langle n\rangle \approx \frac{\langle t\rangle}{\Delta t}$.

Important discrepancies are expected for small $N$ or when the particle starts off near the boundary, since the difference
TABLE III. Values of $\langle n\rangle_{\text {center }}$ for increasing lattice size.

\begin{tabular}{lc}
\hline \hline$N=L \times L$ & $\langle n\rangle_{\text {center }}$ \\
\hline $3 \times 3$ & 1.1782 \\
$5 \times 5$ & 4.7149 \\
$7 \times 7$ & 10.6085 \\
$9 \times 9$ & 18.8595 \\
$11 \times 11$ & 29.4680 \\
$13 \times 13$ & 42.4339 \\
$15 \times 15$ & 57.7573 \\
$17 \times 17$ & 75.4381 \\
\hline \hline
\end{tabular}

between continuum diffusion and on-lattice hopping becomes most pronounced when the number of jumps is small.

Finally, to make contact with the site-specific walk lengths, the "physical coordinates" for the origin of the random walk in terms of lattice coordinates are $x_{0}=(i-1) \Delta \ell$ and $y_{0}=$ $(j-1) \Delta \ell$, where $i, j$ both run from 2 to $L-1$ (the values 1 and $L-1$ are excluded, since they correspond to boundary lattice sites). In particular, when the particle starts from the center of the square one, obtains

$$
\begin{aligned}
\langle n\rangle_{\text {center }} & \approx \frac{\langle t\rangle_{\text {center }}}{\Delta t} \\
& =4 \times 0.07367(L-1)^{2}=0.2947(L-1)^{2} .
\end{aligned}
$$

$\langle n\rangle_{\text {center }}$ can be regarded as the analog of $\left\langle n\left(S_{b}\right)\right\rangle$ provided by the continuum approach. Note that Eq. (28) confirms the linear behavior in $N$ obtained for a square-planar lattice in the large-system limit (cf. the last paragraph in Sec. IV). Displayed in Table III are the results obtained for $\langle n\rangle_{\text {center }}$ by evaluating Eq. (28) for different values of $L$. Comparison with the numerically exact Markov results for $\left\langle n\left(S_{b}\right)\right\rangle$ displayed in the last column of Table II shows that the continuum approach slightly overestimates the actual walk length, but the relative error $\left[\left\langle n_{\text {center }}\right\rangle-\left\langle n\left(S_{b}\right)\right\rangle\right] /\left\langle n\left(S_{b}\right)\right\rangle$ made by the continuum approximation is seen to decrease rapidly with increasing system size.

\section{CONCLUSIONS}

Since the pioneering work of Smoluchowski [28], the study of diffusion-controlled reactions has played a seminal role in understanding the dynamics of reactive processes. Several excellent reviews of the field have been presented (see Refs. [29,30]). The results reported in Refs. [29,30] were derived assuming, for the most part, that the reaction space is spatially homogeneous and classical diffusion theory was invoked. When this theory is augmented by incorporating a nonlinear functional dependence on the concentration of reactants, diverse new phenomena can arise, e.g., "dissipative structures" [31] or "chaotic attractors" [32]. Efforts to understand such processes in spatially inhomogeneous systems have typically proceeded by assuming discrete (lattice) geometries with distributed imperfections, e.g., lacunary regions inaccessible to the diffusing reactant(s). Prominent in this genre of studies have been models based on random or deterministic fractals. By taking advantage of the self-similar properties of the latter class of fractals, the implementation of lattice 
statistical theories has led to a number of exact, analytic results (see the references cited earlier and the discussion below).

The main focus of the present article has been to quantify diffusion-controlled reactive events on a lattice designed to have a fractal-like structure, and to compare the results obtained with processes on a "companion" lattice system free of imperfections or lacunary regions. For both discrete geometries, we consider two scenarios, viz., immobilization of a randomly diffusing reactant at a centrosymmetric reaction center and, second, immobilization of a particle emitted from a centrosymmetric (or other) site at one or more target sites on the boundary of the defined lattice. These studies were complemented by analytic results derived in the continuum limit using classical diffusion theory. A comparison of the results obtained in the lattice and continuum approaches will be given below.

We implemented first the theory of finite Markov processes and calculated one signature of a diffusion-reaction process, the mean walk length of the diffusing particle before being immobilized (trapped) at a reaction center. The numerically exact results obtained using Markov theory are either integers or ratios of integers. The full body of results calculated for the lattices studied here is presented in the Supplemental Material [24].

Displayed in the summary (Table I) are the site-specific walk lengths for diffusive flows from satellite sites to a centrosymmetric trap. Signatures of interest are the mean walk length from all satellite sites to the trap, $\langle n(\mathrm{avg})\rangle$, the mean walk length from satellite sites on the boundary (only) to the trap, $\langle n[\operatorname{avg}(B)]\rangle$, the mean walk length of a random walker from any midpoint site on the boundary to the trap, $\langle n[B(\mathrm{mp})]\rangle$, and from any corner site on the boundary to the trap, $\langle n[B(c)]\rangle$.

In Table II, site-specific walk lengths are given for a random walker from selected satellite sites to a corner trap on the boundary. Here, we specifically restrict $B(\mathrm{mp})$ to be either of the two midpoint sites on the boundary nearest the corner trap, and $B(c)$ to be either of the two corner sites on the boundary nearest the corner trap. The mean walk length to the corner trap for a RW emitted at the source site $S$ is designated $\langle n(S)\rangle$, and the overall average walk length from all satellite sites is $\langle n(\operatorname{avg})\rangle$. Also presented in Table II are RW results calculated for a particle emitted at $S$ but for the limiting case where all $4(L-1)$ sites on the boundary are specified to be deep traps, $\left\langle n\left(S_{b}\right)\right\rangle$.

Qualitatively, one anticipates that lowering the symmetry of the host lattice should result in an increase in the magnitude of all calculated walk lengths. The data recorded in Tables I and II provide a quantitative measure of the consequences of breaking the symmetry of a translationally invariant lattice to one having defined (but not self-similar) lacunary regions. As is evident from the data in Tables I, II, and Tables A1 and A2 in the Supplemental Material [24], the disparity in mean walk lengths becomes more pronounced with increasing spatial extent of the two lattices.

The analytic results presented in this article for random walks on the fractal-like lattice [see Eq. (6) for RWs to a central trap, and Eqs. (7) and (8) and Eqs. (9) and (10) for RWs to the boundary] can be compared with analytic solutions obtained previously for random walks on two deterministic fractals, the Sierpinski "gasket" $[11,14]$ with fractal dimension $d_{f}=\ln 3 / \ln 2 \approx 1.584$, and the Sierpinski "tower" with $d_{f}=$ 2 [12]. The analytic solution for the mean walk length of a particle performing a RW on a finite, planar Sierpinski gasket with a single trap at one vertex is given by

$$
\langle n\rangle^{(\gamma)}=\left[3^{\gamma} 5^{\gamma+1}+4\left(5^{\gamma}\right)-3^{\gamma}\right] /\left(3^{\gamma+1}+1\right),
$$

where $\gamma$ denotes the generation index of the gasket. Embedding the gasket in an arbitrary number $d$ of Euclidean dimensions [12] results in an analytic solution that is structurally similar to Eq. (29), viz.,

$$
\begin{aligned}
\langle n\rangle^{(\gamma)}= & d^{2}\left[(d+1)^{\gamma}(d+3)^{\gamma+1}+(d+2)(d+3)^{\gamma}\right. \\
& \left.-(d+1)^{\gamma}\right] /\left\{(d+2)\left[(d+1)^{\gamma+1}+d-1\right]\right\} .
\end{aligned}
$$

Comparison of the results obtained for the fractal-like lattice and those obtained for the deterministic fractals, the Sierpinski gasket, and tower shows that the functional dependence is consistently algebraic.

The algebraic dependence on system size for the fractallike lattice stands in contrast to the transcendental $N \ln N$ dependence found by Montroll and Weiss for regular, periodic $d=2$ dimensional lattices of uniform coordination with a centrosymmetric trap [Eq. (4)]. We showed in Sec. V that this functional dependence of trapping on system size for translationally invariant, discrete lattices is also found if one passes to the continuum limit and mobilizes classical diffusion theory [see Eqs. (16)].

For the second scenario considered in this article, the continuит theory presented in Sec. VI resulted in an expression for the trapping time for a particle initialized in a centrosymmetric region and immobilized eventually at the system's boundary. It was proved that the trapping time grows linearly with the system size $N$ [see Eq. (28)]. As for the Markovian theory, we showed in Sec. IV that for a particle emitted at the centrosymmetric site $S$, undergoing a random walk until immobilized at (any site of) the boundary, the trapping time (as gauged by the overall mean walk length; see Sec. II) also behaves as $\left\langle n\left(S_{b}\right)\right\rangle \sim N$, a result which stands in contrast to the behavior found for the fractal-like lattice where, in the limit of large-system size, $\left\langle n\left(S_{b}\right)\right\rangle \sim N \sqrt{ } N$ [see Eq. (10)].

Further insight on the analytic structure of the results obtained for the fractal-like lattice follows from the result obtained in Ref. [21] for RWs on a $d=1$ dimensional Euclidean lattice of $N=\mathrm{L}$ sites; there it was proved that the mean walk length to a single deep trap is given exactly by $\langle n\rangle=(1 / 6) L(L+1)$.

The fractal-like lattice introduced here is of Euclidean dimension $d=2$, but pathways through the lattice to one or more deep traps are restricted by (effectively) $d=1$ channels, so in hindsight it is perhaps not surprising that the dependence of the mean walk length on system size for both trapping scenarios is algebraic.

Finally, we survey problems to which the results reported in this article can be applied in future work. Clearly, the vast literature on diffusion of atoms on metal surfaces and, especially, cross-channel diffusion [33], provides a wealth of problems to consider. The synthesis of novel organic molecules, and subsequent studies of surface-assisted selfassembly of these molecules on metal surfaces, has led to the design of nanostructures with remarkable functionalities 


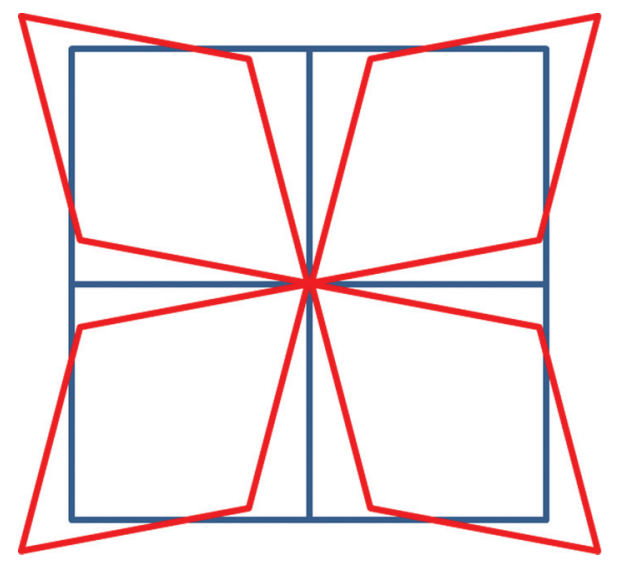

FIG. 4. (Color) Boundary of a $5 \times 5$ square-planar lattice (in blue) with a centrosymmetric trap. Boundary of four $3 \times 3$ quadrilateral ("rhombus shaped") lattices (in red), each with a corner trap.

and aggregation kinetics [34]. In both cases, the supporting templates are likely to have imperfections, e.g., terraces, ledges, kinks, etc., suggesting that a quantitative assessment of the influence on the dynamics owing to, for example, the class of spatial inhomogeneities considered in the present article would be of value.

Two quite different problems for which intuition suggests a plausible, qualitative interpretation but for which (exact) quantitative estimates are as yet unavailable will be discussed here. The first problem pertains to pattern formation in morphogenesis, and the second to radio-halo formation in minerals.

Consider the geometries displayed in Fig. 4. The figure in blue represents the boundary of a $5 \times 5$ square-planar lattice with a centrosymmetric trap. If a nutrient is injected into the $5 \times 5$ lattice, and engages in a random walk before being trapped at the target site, the overall mean walk length for the random walker before trapping is $\langle n(\operatorname{avg})\rangle=31.67$. See Table I.

Ratio $(3 / 5)=\langle n(\operatorname{avg})\rangle(3 \times 3$ rhombus with a corner trap $) /\langle n(\operatorname{avg})\rangle(5 \times 5$ square - planar lattice with a centrosymmetric trap $)$

$$
=21.9375 / 31.6667=0.693 \text {. }
$$

Similar constructions give

$$
\begin{aligned}
\text { Ratio }(5 / 9) & =88.6900 / 130.6045=0.679, \\
\text { Ratio }(7 / 13) & =212.3538 / 310.6494=0.684, \\
\text { Ratio }(9 / 17) & =400.0071 / 579.5265=0.690 .
\end{aligned}
$$

Surprisingly, the gain in efficiency owing to morphogenetic growth (foliation of template geometry [35]) is found to be essentially a constant, in the range $0.68-0.69$. For the fractallike lattice, the range is $0.56-0.57$, the difference in estimates $(\sim 15 \%)$ reflecting the designed, penniveined pattern.

Successive stages of foliation can be envisioned starting with the $17 \times 17$ square-planar template. In the first stage, one would generate four $9 \times 9$ leaves; in the second stage, each $9 \times 9$ leaf would generate four $5 \times 5$ leaves, and in the third stage, each $5 \times 5$ leaf would generate four $3 \times 3$ leaves. At
The $5 \times 5$ square-planar lattice can be parsed into four quadrants defined by the intersection at the centrosymmetric site of one horizontal and one vertical $C_{2}$ axis; each quadrant is a $3 \times 3$ square-planar lattice. If the vertical axis and the horizontal axis in the upper-right quadrant are rotated infinitesimally to the right and left, respectively, keeping the centrosymmetric trap fixed, the resulting, slightly distorted, $3 \times 3$ lattice forms a rhombus; the figure in red in the upper-right-hand quadrant shows the boundary of the rhombus.

Considering all four quadrants, each undergoing a similar, (essentially) area-preserving diffeomorphic distortion, the resultant antenna geometry of four rhombuses, each sharing a common corner trap (the origin of the original $5 \times 5$ lattice) is shown in Fig. 3. In this case, a nutrient injected into any one of the lattice quadrants reaches the target site after a total of $\langle n(\operatorname{avg})\rangle=21.94$ steps. See Table II.

Taking the mean walk length as a measure of reaction efficiency, a nutrient injected into a $(5 \times 5)$ square-planar antenna at any receptor site will be trapped at the centrosymmetric target site in 31.7 steps, whereas if injected into any one of the four rhombuses defining a new antenna, the mean walk length before trapping at the corner site is 21.9 steps. Since the transformation disconnects the four quadrants, effectively reducing the unit lattice size, one anticipates that this morphology will lead to a more efficient diffusion-reaction process. The calculations reported here put this qualitative understanding on a quantitative footing.

A similar program can be carried out starting from a $9 \times 9$ square-planar lattice with a centrosymmetric trap to generate four $5 \times 5$ rhombuses sharing a common corner trap; starting from a $13 \times 13$ square-planar lattice to generate four $7 \times 7$ rhombuses sharing a common corner trap; and starting from a $17 \times 17$ square-planar lattice to generate four $9 \times 9$ rhombuses sharing a common corner trap. From the mean walk-length data given in the tables, we can compare quantitatively the reaction efficiency in each new morphology relative to the precursor morphology. In the example given above, we define the ratio each stage of foliation there would be an essentially constant gain in reaction efficiency. This gain in "signal processing" is similar to that found in electrical antenna networks, except that in the latter there is a dependence on the wavelength of the incoming signal.

A second problem that can be addressed using the approach taken in this article is the occurrence of pleochroic halos in brown micas, first reported by Joly in 1907, corroborated shortly thereafter by Rutherford, and analyzed in a joint publication in 1913 [36]. The phenomenon is described in the first sentence of [36]: "It is now well established that the minute circular marks seen in sections of certain coloured rock minerals-notably the coloured micas-are due to the effects produced by the alpha radiation of a central radioactive particle." And, "the halo is in every case the result of the integral actions of rays emitted since a very remote period." 
The lacunary regions in the fractal-like lattice displayed in Fig. 1(b) can be regarded as mineral grain boundaries which obstruct the trajectory of an $\alpha$ particle emitted at the source $S$ in its passage through the lattice. To make contact with the temporal estimate of Joly and Rutherford on the age of radio halos, one needs to specify in our lattice statistical model a reliable estimate of the mean time between displacements of the $\alpha$ particle. Whatever the estimate, once established, a glance at the data for $\langle n(S)\rangle$ and $\left\langle n\left(S_{b}\right)\right\rangle$ in Table II for a defect-free lattice versus the fractal-like one shows that any estimate of the time required before localization of the $\alpha$ particle at a distant site based on a lattice statistical theory [or molecular dynamics (MD) simulation] of trapping on a defect-free lattice or based on a Fickian continuum theory in which all structural details are suppressed, will underestimate the integrated trapping time (and "age" of the radio halo) by (at least) an order of magnitude.

Finally, it is worth pointing out the generic interest of studying other random walk properties of the fractal-like lattice, such as the behavior of higher-order moments of the walk length or simply the mean square displacement of a random walker undergoing obstructed diffusion. A priori one would expect the onset of anomalous subdiffusion due to the stalling events induced by the lattice. The underlying question is to what extent such effects could be captured in the framework of an effective description in terms of a continuous time random walk (CTRW)/fractional diffusion equation [37] or a generalized Langevin equation [38], and, if answered in the affirmative, what the relation between the lattice geometry and the characteristic exponents in those models looks like.

\section{ACKNOWLEDGEMENTS}

The authors would like to thank S. B. Yuste, J. Wm. Turner, and S. Lelic for valuable insights on aspects of the problem discussed here. Technical assistance has been provided by P. Panagiotopolous. This study was initiated while J.J.K. was in residence at the Beckman Institute, and he is grateful to H. B. Gray for his kind hospitality. Financial support for R.A.G.-L. was provided by the Hirsh Research Initiation Grant, the Howard Hughes Medical Institute Research Program, and the Summer Undergraduate Research Program from Pomona College. E.A. gratefully acknowledges financial support from Ministerio de Ciencia y Tecnología (Spain) through Grant No. FIS2010-16587 (partially funded by FEDER funds) as well as additional financial support from Junta de Extremadura through Grant No. GRU10158.
[1] B. O’Shaughnessy and I. Procaccia, Phys. Rev. A 32, 3073 (1985).

[2] S. Havlin and D. Ben-Avraham, Adv. Phys. 36, 695 (1987).

[3] C. Van den Broeck, Phys. Rev. Lett. 62, 1421 (1989).

[4] J.-P. Bouchaud and A. Georges, Phys. Rep. 195, 127 (1990).

[5] C. van den Broeck and V. Balakrishnan, Ber. Bunsenges. Phys. Chem. 95, 342 (1991).

[6] V. Balakrishnan, Mater. Sci. Eng., B 32, 201 (1995); C. P. Haynes and A. P. Roberts, Phys. Rev. E 78, 041111 (2008).

[7] J. J. Kozak, J. Stat. Phys. 101, 405 (2000).

[8] B. M. Hambly, Ann. Probab. 25, 1059 (1997).

[9] M. T. Barlow and B. M. Hambly, Ann. Inst. H. Poincaré 33, 531 (1997).

[10] B. M. Hambly, T. Kumagai, S. Kuzuoka, and X. Y. Zhou, J. Math. Soc. Jpn. 52, 373 (2000).

[11] J. J. Kozak and V. Balakrishnan, Phys. Rev. E 65, 021105 (2002).

[12] J. J. Kozak and V. Balakrishnan, Int. J. Bifurcation Chaos Appl. Sci. Eng. 12, 2379 (2002).

[13] M. A. Bab, G. Fabricius, and E. V. Albano, J. Chem. Phys. 128, 044911 (2008).

[14] J. L. Bentz, J. W. Turner, and J. J. Kozak, Phys. Rev. E 82, 011137 (2010).

[15] S. Weber, J. Klafter, and A. Blumen, Phys. Rev. E 82, 051129 (2010).

[16] S. Wu, Z. Zhang, and G. Chen, Eur. Phys. J. B 82, 91 (2011).

[17] B. Meyer, E. Agliari, O. Benichou, and R. Voituriez, Phys. Rev. E 85, 026113 (2012).

[18] G. J. Staten, M. K. Mucho, and J. J. Kozak, Langmuir 1, 443 (1985).

[19] G. A. Somorjai, K. M. Bratlie, M. O. Montano, and J. Y. Park, J. Phys. Chem. B 110, 20014 (2006).

[20] J. Jung, H.-J. Shin, Y. Kim, and M. Kawai, J. Am. Chem. Soc. 133, 6142 (2011).
[21] E. W. Montroll, in Proceedings of the Symposium on Applied Mathematics (American Mathematical Society, Providence, RI, 1964), Vol. 16; E. W. Montroll and G. H. Weiss, J. Math. Phys. 6, 167 (1965); E. W. Montroll, ibid. 10, 753 (1969).

[22] (a) E. W. Montroll and K. E. Shuler, Adv. Chem. Phys. 1, 361 (1958); (b) G. Kemeny and J. L. Snell, Finite Markov Chains (Van Nostrand, Princeton, NJ, 1960); (c) G. H. Weiss, Aspects and Application of the Random Walk (North-Holland, Amsterdam, 1994).

[23] (a) C. A. Walsh and J. J. Kozak, Phys. Rev. Lett. 47, 1500 (1981); Phys. Rev. B 26, 4166 (1982); (b) P. A. Politowicz and J. J. Kozak, ibid. 28, 5549 (1983); (c) J. J. Kozak, Adv. Chem. Phys. 115, 245 (2000).

[24] See Supplemental Material at http://link.aps.org/supplemental/ 10.1103/PhysRevE.89.032147 for site-specific walk lengths to a centrosymmetric sink (trap) and to a corner trap on a regular square-planar lattice of $N$ sites and a companion, fractal-like lattice of $N$ sites.

[25] The coefficients $A_{1}, A_{2}, A_{3}$ are reported in Ref. [21]; the coefficient $A_{4}$ was (re)calculated in W. Th. Den Hollander and P. W. Kasteleyn, Physica A 112, 523 (1982); subsequent numerical calculations confirmed their result [P. A. Politowicz and J. J. Kozak, Langmuir 4, 305 (1988)].

[26] K. Knopp, Infinite Sequences and Series (Dover, New York, 1956); C. N. Moore, Summable Series and Convergence Factors (Dover, New York, 1966); L. B. W. Jolley, Summation of Series, 2nd ed. (Dover, New York, 1961).

[27] (a) H. S. Carslaw and J. C. Jaeger, Conduction of Heat in Solids, 2nd ed. (Clarendon, Oxford, U.K., 2000); (b) S. Redner, A Guide to First Passage Processes (Cambridge University Press, New York, 2001).

[28] M. Smoluchowski, Z. Phys. Chem. 92, 129 (1917). 
[29] S. A. Rice, Diffusion-Limited Reactions (Elsevier, Amsterdam, 1985); E. Kotomin and V. Kuzovkov, Modern Aspects of Diffusion-Controlled Reactions (Elsevier, Amsterdam, 1996).

[30] L. G. Harrison, Kinetic Theory of Living Pattern (Cambridge University Press, Cambridge, U.K., 1993); R. Erban and S. J. Chapman, Phys. Biol. 6, 046001 (2009).

[31] G. Nicolis and I. Prigogine, Self-Organization in Nonequilibrium Systems (Wiley, New York, 1977).

[32] H. Haken, Advanced Synergetics (Springer, Berlin, 1993).

[33] See, for example, G. Antczak and G. Ehrlich, Surf. Sci. Rep. 62, 39 (2007).
[34] S. Mohnani and D. Bonifazi, Coord. Chem. Rev. 254, 2342 (2010); T. R. Cook, Y.-R. Zheng, and P. J. Stang, Chem. Rev. 113, 734 (2013); G. Nicolis, J. J. Kozak, and C. Nicolis, Mol. Phys. 110, 395 (2012).

[35] D. W. Thompson, On Growth and Form (Dover, New York, 1992), Chap. 17.

[36] J. Joly and E. Rutherford, Philos. Mag. 25, 644 (1913).

[37] See, e.g., R. Metzler and J. Klafter, J. Phys. A: Math. Gen. 37, R161 (2004) and references therein.

[38] See L. C. Lapas, R. Morgado, M. H. Vainstein, J. M. Rubí and F. A. Oliveira, Phys. Rev. Lett. 101, 230602 (2008) and references therein. 